\title{
Timing of urgent cardiac and aortic surgery in Covid-19 positive patients: Earliest safe period for surgery.
}

\author{
Ana Lopez-Marco ${ }^{1}$, Azhar Hussain ${ }^{1}$, Martin Yates ${ }^{1}$, Damian Balmforth ${ }^{1}$, Rakesh Uppal ${ }^{1}$, \\ and Aung $\mathrm{Oo}^{1}$ \\ ${ }^{1}$ Saint Bartholomew's Hospital
}

May 28, 2020

\begin{abstract}
OBJECTIVES Surgery in COVID-19 patients carries a significant mortality and morbidity but the appropriate waiting period before a surgical intervention after recovering from COVID-19 is not known. We aim to determine the safety of deferring urgent surgery and to identify the earliest safe period for surgery by analysing our experience in patients who underwent cardiac surgery after recovering from COVID-19. METHODS Analysis of all patients undergoing cardiac surgery in our centre during March and April 2020, focusing in those who tested positive for COVID-19 in the pre-operative screening. RESULTS Four patients tested positive for COVID-19 during the preoperative screening. Two of them also showed radiological signs of COVID-19 lung disease. We delayed surgery until they tested negative and the radiological changes improved. None of the patients suffered a deterioration of their cardiac symptoms that mandated expediting the surgeries. As a result of waiting until COVID-19 resolution, all our patients survived the urgent cardiac surgery without developing any COVID-19-related complications. CONCLUSIONS From our experience, defering urgent cardiac surgery in patients with confirmed COVID-19 is safe. Operating as early as 4 days after a negative swab does not increase mortality and morbidity.
\end{abstract}

\section{MAIN TEXT}

\section{INTRODUCTION}

The novel Coronavirus (SARS-CoV-2) cause of the clinical syndrome COVID-19, associates a high incidence of severe acute respiratory syndrome requiring invasive mechanical ventilation. Due to the rapid expansion of the infection and the disease, the World Health Organisation (WHO) declared COVID-19 a global pandemic on the 11th March 2020.[1]

Since then an important restructuring of the healthcare system has taken place and all elective surgical activity has been put on hold to reallocate intensive care beds preferentially to COVID-19 patients.

Before the pandemic was declared and screening protocols were not fully established, surgical activity continued without personal protective equipment (PPE) measures which is now the standard of care universally [2]. Several reports, mainly from China, describe high rate of mortality (up to 25\%) and morbidity (over $40 \%$ need for prolonged mechanical ventilation) in patients who underwent surgical procedures and were diagnosed of COVID-19 during the peri-operative period [3,4]. In our own experience, the development of COVID-19 in the immediate postoperative period after cardiac surgery carries a mortality up to $44 \%$.

Since the declaration of the Pandemic in London, we concentrated in improving preoperative screening measures and defer cardiac surgery in positive patients when time permitting. Some symptomatic patients were treated with alternative treatment options e.g.PCI or TAVI.

For urgent conditions, there is an opportunity for deferring surgery until COVID-19 conversion from positive to negative, while monitoring the patients for their cardiac condition closely in the hospital environment and 
revisit the timing of the operation if there is a clinical change. For emergency conditions, however, there may not be enough time for COVID-19 screening, and a balanced decision should be taken weighing the cardiac-related mortality against the COVID-19-related mortality if proven positive.

It is, however, uncertain how to predict when a COVID-19 positive patient will become negative (and not just a false negative) and the duration of appropriate waiting period between the negative result and the surgical intervention.

We aim to identify when is the earliest safe period for surgery by analysing our experience in patients who underwent cardiac surgery after recovering from COVID-19.

\section{$M E T H O D S$}

Analysis of all patients undergoing elective, urgent or emergency cardiac surgery at Barts Heart Centre from $1^{\text {st }}$ March to $30^{\text {th }}$ April 2020, focusing the review in those who tested positive for COVID-19 in the PCR-RNA analysis of nasal and throat swabs carried out as a part of the pre-operative work-up.

Our local COVID-19 screening protocol consists of requirement for two consecutive negative nasopharyngeal swabs for polymerase chain reaction for ribonucleic acid (PCR-RNA) analysis of Covid-19, before accepting the patient for surgery. [5]

We also request a non-contrasted $\mathrm{CT}$ of the chest to assess changes in the lung parenchyma suggested of COVID-19 disease (e.g. ground-glass opacities, interstitial infiltrates). As adjuncts, we also record lymphocyte count and Lactate Dehydrogenase (LDH) levels [5-8].

Only when time is not permitting due to clinical emergency, the screening results are not awaited; instead the patients are considered COVID-19 positive and treated accordingly with the necessary precautions during the surgery and the perioperative period until the results of a deep tracheal swab and bronchial aspirate come back negative.

Data was obtained retrospectively from the individual clinical records and the national cardiac surgery database. Individual consent from patients was waived by the Ethics Institutional Board since there is no identifiable data.

\section{RESULTS}

Four patients were found to be COVID-19 positive in the preoperative screening. As none of the patients posed a clinical emergency, the surgery was postponed until COVID-19 negative results were obtained.

All the patients were male, their mean age was 58.2 years ( $45-68$ years) and all of them were admitted to our unit with urgent conditions (acute myocardial infarction, native valve infective endocarditis, rapidly expanding aortic aneurysm and infected previous ascending aortic graft with a communicating fistula to the sternal wound). (Table 1)

All patients were screened for COVID-19 as part of our previously described protocol on the first day of admission and the screening swabs were repeated after 5 days, and subsequently every 48 hours until the desired two negative tests were obtained, and the patients were listed for urgent surgery.

Two patients tested negative on the first repeated swab while the other two have four subsequent positive swabs before becoming negative. The mean delay to surgery was 17.5 days ( $5-31$ days). (Table 1 )

Two of the patients showed radiological changes in the non-contrasted CT chest, described as diffuse groundglass opacities, predominantly on the lower lobes and suggestive of COVID-19 pneumonitis. These two patients were closely monitored with serial CXR showing resolution of the infiltrates in a week time. (Table 2)

None of the patients developed pyrexia $\left(>38^{\circ} \mathrm{C}\right)$ and only one patient was categorized as having COVID-19 symptoms, consistent on breathlessness, but this could also be attributed to his presentation with acute coronary syndrome and heart failure in addition to the suggested pneumonitis for COVID-19. (Table 2) 
Blood test analysis revealed non-specific changes. The highest white blood cell count at presentation was normal (median $\left.7.5 \times 10^{9} \mathrm{c} / \mathrm{L},\left(5.6-28.3 \times 10^{9} \mathrm{c} / \mathrm{L}\right)\right)$ except for the patient presenting with acute coronary syndrome and heart failure. The lymphocyte count varied from 0.5 to $1.0 \times 10^{9} \mathrm{c} / \mathrm{L}$ and the LDH and CRP values were deemed unreliable due to the concomitant diagnosis of ongoing infection (endocarditis and aortic graft infection) or acute inflammatory events (acute coronary syndrome and expanding aneurysm). (Table 2)

All patients underwent surgery once tested negative for COVID-19, although PPE precautions were taken during the operation and postoperative period. The repeated swabs after surgery (taken between postoperative days 1-9) were negative for all patients.

None of the patients developed any respiratory complications related to COVID-19, and they were all extubated uneventfully on the same day of surgery or the following two days depending on the nature of the original operation. Mean mechanical ventilation times were 12.5 hours (6-24 hours) and the mean of stay in ITU was 3.5 days $(3-4$ days). (Table 3 )

All the patients are alive and two of them have already been discharged home well (mean total hospital stay 24.5 days (18 - 31 days), mean postoperative hospital stay 7 days ( $5-9$ days), while the other two remain in hospital to complete a six-week regime of intravenous antibiotics due to their original diagnosis. (Table 3)

\section{DISCUSSION}

The accuracy of the COVID-19 diagnostic tests is still suboptimal, with a high percentage of false negatives on the PCR-RNA analysis from nasopharyngeal swab samples [9]. Also, there are cases where the individuals with a previous negative test, become positive again in subsequent tests without any associated clinical deterioration that would help in predicting this [10].

The ideal convalescence period after having tested positive for COVID-19 it is also unknown, especially in the absence of symptoms or abnormalities in the chest imaging modalities.

We know from previous reports out of China [3,4] and our own experience, that patients who develop COVID-19 during the immediate postoperative period, regardless of the nature of the surgical procedure, have a bad prognosis, with high mortality and morbidity related to respiratory complications.

In the current era, when all the elective surgical procedures have been paused to prioritise intensive care resources to accommodate the COVID-19 patients, we have concentrated in treating urgent and emergency cardiac and aortic surgery cases. We have established a rigorous COVID-19 screening protocol following international recommendations, including the combination of questionnaire for COVID-19-related symptoms, two negative nasopharyngeal swabs, clear lung fields on a non-contrasted CT chest and the absence of lymphopenia or excessively elevated LDH levels [5].

In emergency cases there is no option to wait for the swab results, hence the surgical indication outweighs the risk of COVID-19 infection especially in the patients with no COVID-19-related symptoms and pulmonary infiltrates on the CT chest.

However, in the urgent cases, with at least $24-48$ hours margin to wait for the screening results, we are now facing a proportion of patients who are COVID-19 positive with mild symptoms or even not symptomatic at all. Despite that, all patients are treated as if they were positive and all the universal PPE precautions taken [5].

To date there is little knowledge of the predictors to determine the earliest safe time to plan an operation, in patients who may still be infectious and/or with potential for reactivation in the immediate postoperative period, with potential catastrophic consequences for their respiratory system after having been exposed to the cardiopulmonary bypass with the subsequent systemic inflammatory response. 
For this reason, we believe it is important to manage each case individually, and when time permitting, to wait until the viral load and the radiological infiltrates (if any) have resolved.

In our experience, the close monitoring in a hospital environment, where the patients were kept in isolation and only interacting with full-PPE equipped healthcare professionals, allowed us to postpone the urgent surgery until they became COVID-19 negative. With those measures in place, we achieved a good outcome for all of them, without any COVID-19-related complications in the postoperative period and a 100\% survival rate.

Further studies on a larger volume of patients, like the CovidSurg international multicentre study, will help to increase to understand the impact of COVID-19 during the peri-operative period and add some clarity and recommendations to the optimal timing for offering surgery in patients recovered from COVID-19.

\section{CONCLUSIONS}

From our experience with this small series of patients, defering urgent cardiac surgery in clinically stable patients with confirmed COVID-19 while under close monitoring is safe and allowed to perform surgery with favourable outcomes. Operating as early as 4 days after negative swab does not increase mortality and morbidity.

Further studies on larger volume of patients are required to increase the understanding of the impact of COVID-19 during the peri-operative period, the implications of operating in positive patients and the recovery period recommended before attempting surgical treatment when time permits.

TABLES

\begin{tabular}{lllllll}
\hline Patient & Sex & Age & Diagnosis & Date of hospital admission & Date of COVID diagnosis & Nu \\
\hline 1 & M & 68 & Aortic valve endocarditis & $23^{\text {rd }}$ March & $23^{\text {rd }}$ March & 4 \\
2 & M & 61 & STEMI & $13^{\text {th }}$ April & $13^{\text {th }}$ April & 1 \\
3 & M & 59 & Expanding DTA aneurysm & $29^{\text {th }}$ March & $29^{\text {th }}$ March & 1 \\
4 & M & 45 & Infected aortic graft & $2^{\text {nd }}$ April & $3^{\text {rd }}$ April & 4 \\
\hline
\end{tabular}

Table 1

\begin{tabular}{|c|c|c|c|c|c|c|c|}
\hline Patient & $\begin{array}{l}\text { COVID } \\
\text { symptoms }\end{array}$ & Pyrexia & $\begin{array}{l}\text { Highest } \\
\text { White } \\
\text { Blood cells } \\
\text { count }\end{array}$ & $\begin{array}{l}\text { Lowest } \\
\text { Lympho- } \\
\text { cyte } \\
\text { count }\end{array}$ & $\begin{array}{l}\text { Highest } \\
\text { LDH Value }\end{array}$ & $\begin{array}{l}\text { Highest } \\
\text { CRP value }\end{array}$ & $\begin{array}{l}\text { CT } \\
\text { changes } \\
\text { Suggested } \\
\text { of COVID }\end{array}$ \\
\hline 1 & No & No & 6.7 & 0.5 & 350 & 109 & No \\
\hline 2 & SOB & No & 28.3 & 1.0 & $\mathrm{~N} / \mathrm{A}$ & 114 & Yes \\
\hline 3 & No & No & 8.3 & 1.6 & 177 & 103 & No \\
\hline 4 & No & No & 5.6 & 0.6 & $\mathrm{~N} / \mathrm{A}$ & 74 & Yes \\
\hline
\end{tabular}

Table 2

\begin{tabular}{lllll}
\hline Patient & Surgery & COVID status after surgery & Mechanical ventilation times & Length of ITU adn \\
\hline 1 & AVR & Negative & 8 & 3 \\
2 & CABG & Negative & 6 & 3 \\
3 & DTA replacement & Negative & 24 & 4 \\
4 & AA replacement & Negative & 12 & 4 \\
\hline
\end{tabular}


Table 3

\section{LEGENDS}

Table 1. Demographics, cardiac diagnosis and details of the nasopharyngeal COVID-19 swabs screening. DTA: descending thoracic aorta; STEMI: ST elevation myocardial infarction.

Table 2. Presence of COVID-19 symptoms and analysis of screening adjuncts: blood rest rruslts including white blood cell and lymphocyte counts, Lactate dehydrogenase (LDH) and C- reactive protein (CRP) values and suggestive changes oh the non-contrasted chest computed tomography (CT). SOB: sorthness of breath. N/A (Non-applicable) means the result was not available due to heamolysed sample.

Table 3. Postoperative outcomes including COVID-19-respiratory related complications and length of intensive care (ITU) stay. AA: ascending aorta, AVR: aortic valve replacement, DTA: descending thoracic aorta.

\section{REFERENCES}

1. World Health Organization. WHO announces COVID-19 outbreak a pandemic. World Heal. Organ. 2020;:3.http://www.euro.who.int/en/health-topics/health-emergencies/coronaviruscovid-19/news/news/2020/3/who-announces-covid-19-outbreak-a-pandemic.

2. NHS England. Next Step on NHS response to COVID-19. 2020.https://www.england.nhs.uk/coronavirus/wp-content/uploads/sites/52/2020/03/urgent-nextsteps-on-nhs-response-to-covid-19-letter-simon-stevens.pdf (accessed 23 Apr 2020).

3. Cai Y, Hao Z, Gao Y, Ping W et al. Coronavirus disease 2109 in the perioperative period of lung resection: a brief report from a single thoracic surgery department in Wuhan, People's Republic of China. J Thorac Oncol. 2020 Apr 11:S1556-0864(20)30298-7.

4. Lei S, Jiang F, Su W, Chen C et al. Clinical characteristics and outcomes of patients undergoing surgeries during the incubation period of COVID-19 infection. EClinicalMedicine. 2020 Apr 5:100331

5. SCTS. St Bartholomew's Hospital Theatre Standard Operating Protocol for COVID-19. 2020.https://scts.org/wp-content/uploads/2020/04/St-Bartholomews-Theatre-SOP-for-COVID19-8th-April-2020.pdf (accessed 23 Apr 2020).

6. Hope MD, Raptis CA, Shah A, et al. A role for CT in COVID-19? What data really tell us so far. Lancet Published Online First: 2020.

7. Han Y, Zhang H, Mu S, et al. Lactate dehydrogenase, a Risk Factor of Severe COVID-19 Patients. medRxiv Published Online First: 2020.

8. Tan L, Wang Q, Zhang D, et al. Lymphopenia predicts disease severity of COVID-19: a descriptive and predictive study. Signal Transduct. Target. Ther. 2020.

9. Li Y, Yao L, Li J, et al. Stability issues of RT-PCR testing of SARS-CoV-2 for hospitalized patients clinically diagnosed with COVID-19. J Med Virol Published Online First: 2020.

Xing Y, Mo P, Xiao Y, Zhao O et al. Post-discharge surveillance and positive virus detection in two medical staff recovered from coronavirus disease 2019 (COVID-19), China, January to February 2020. Euro Surveill, 2020 Mar 12: 25(10):2000191. 